\title{
A novel one-step approach for the construction of yeast surface display Fab antibody libraries
}

\author{
Simon Rosowski', Stefan Becker², Lars Toleikis², Bernhard Valldorf ${ }^{3}$, Julius Grzeschik¹, Deniz Demir², \\ Iris Willenbücher², Ramona Gaa², Harald Kolmar', Stefan Zielonka ${ }^{2 *}$ and Simon Krah²*
}

\begin{abstract}
Background: Yeast surface display (YSD) has proven to be a versatile platform technology for antibody discovery. However, the construction of antibody Fab libraries typically is a tedious three-step process that involves the generation of heavy chain as well as light chain display plasmids in different haploid yeast strains followed by yeast mating.

Results: Within this study, we aimed at implementing a focused Golden Gate Cloning approach for the generation of YSD libraries. For this, antibodies heavy and light chains were encoded on one single plasmid. Fab display on yeast cells was either mediated by a two-directional promoter system (2dir) or by ribosomal skipping (bicis). The general applicability of this methodology was proven by the functional display of a therapeutic antibody. Subsequently, we constructed large antibody libraries with heavy chain diversities derived from CEACAM5 immunized animals in combination with a common light chain. Target-specific antibodies from both display systems were readily obtained after three rounds of fluorescence activated cell sorting. Isolated variants exhibited high affinities in the nanomolar and subnanomolar range as well as appropriate biophysical properties.
\end{abstract}

Conclusion: We demonstrated that Golden Gate Cloning appears to be a valid tool for the generation of large yeast surface display antibody Fab libraries. This procedure simplifies the hit discovery process of antibodies from immune repertoires.

Keywords: Golden Gate Cloning, Yeast surface display, Fab fragment, Antibody discovery

\section{Background}

During recent decades, Yeast Surface Display (YSD) has proven to be a versatile technology for antibody engineering and hit discovery [1-7]. Pioneered by Boder and Wittrup in 1997, the genotype-phenotype coupling principle was initially demonstrated with an affinity maturation process of a FITC binding single chain antibody fragment $(\mathrm{scFv})$ [1]. To this date, many other applications, for instance antibody stability- and $\mathrm{pH}$-engineering $[3,8-10]$, antigen based selection of antibody repertoires from naïve [11] and synthetic sources $[6,12,13]$ as well as

\footnotetext{
*Correspondence: Stefan.Zielonka@merckgroup.com;

Simon.Krah@external.merckgroup.com

${ }^{2}$ Protein Engineering and Antibody Technologies, Merck KGaA,

Frankfurter Strasse 250, 64293 Darmstadt, Germany

Full list of author information is available at the end of the article
}

from immunized animals were reported [2, 4]. Moreover, many non-conventional antibodies and scaffold proteins were engineered using this platform technology $[6,13-$ 15]. Compared to other cell based selection systems like bacterial and phage display, one beneficial feature of YSD is the use of the eukaryotic expression host S. cerevisiae for the production of the displayed protein [1]. The presence of sophisticated quality control machineries residing in the endoplasmatic reticulum and Golgi apparatus might enable a more precise manufacturing of complex proteins in comparison to the prokaryotic host $E$. coli [1]. In addition, it's compatibility with fluorescence activated cell sorting (FACS) enables real-time and on-line analysis as well as a fine discrimination of variants exhibiting different prescribed properties such as affinity or stability. 
In the context of antibody discovery, the display of a variety of antibody formats are described in literature, ranging from simple antibody fragments like scFvs over Fab-fragments to full length IgGs $[1,4,5,16]$. The classical approach for the display of e.g. Fab-fragments relies on the individual generation of heavy and light chain plasmids encoding regions $\mathrm{VH}-\mathrm{CH} 1$ and $\mathrm{VL}-\mathrm{CL}$, respectively, via homologous recombination in haploid yeast strains. Afterwards, these haploid yeast cells can be combined into diploid cells that display functional Fabs on their surface by yeast mating $[2,5]$. In the most common experimental setting, the surface display of the antibody variant is achieved by genetic fusion of the heavy chain segment to Aga2p, a cell surface-exposed protein that is anchored together with Agalp in the yeast cell wall [1]. Upon co-expression of the light chain, assembly of the heterodimeric heavy and light chain fragment occurs leading to cell-surface exposed Fab [2, 5]. Although, this technology allows for the efficient generation of large antibody libraries, the multi-step process of library generation is tedious and time-consuming.

In 2008, a novel cloning technology was described, referred to as Golden Gate Cloning [17]. This cloning strategy has its origins in 1996, when it was shown that multiple DNA fragments can be cloned into a plasmid by the use of type IIs restrictions enzymes and T4 DNA ligase $[18,19]$. Type IIs restriction enzymes are able to cleave outside of their recognition site, resulting in a DNA overhang which can be composed of any nucleotide sequence. Marillonnet et al. designed the cleavage sites and the resulting overhangs of two DNA fragments in a way that both digested fragments were ligated to a product in a seam-less manner [17]. This allowed for subcloning in a single step and a single tube with a cloning efficacy close to $100 \%$. A major advantage of this cloning method is the independence of the enzyme recognition site from the gene of interest and that the recognition site can be designed to be eliminated during the restriction. In addition, the cleavage site overhangs can be composed of different distinct sequences (herein termed signature sequences) enabling directional cloning of several
DNA fragments and preventing religation of the respective vectors [17]. Consequently, it was shown that up to ten different fragments can be assembled in a defined order by the generation of shuffling libraries, which can improve the outcome of library selection as in the case of trypsinogen variants displaying higher production titers compared to the wild-type protein [20].

In this work, we present a novel one-step Golden Gate Cloning approach for the generation of YSD Fab libraries enabling the simultaneous introduction of heavy chain and light chain variable regions into one single display vector. We demonstrate the versatility of this cloning technology for YSD by designing two different display strategies. In the two-directional system (2dir), expression of the heavy chain is enabled under the control of the Gal1 promoter whereas light chain expression is facilitated via the Gal10 promoter (Fig. 1a). In the bicistronic system (bicis), Fab display is mediated by ribosomal skipping (Fig. 1b) [21, 22]. We show that large antibody libraries with more than $10^{8}$ unique clones can readily be constructed by applying the herein presented approach. Furthermore, we prove that high-affinity antibodies can be isolated from such libraries using CEACAM5 immunized animals as a source of diversity. CEACAM5 [Carcinoembryonic antigen (CEA)] is a protein, expressed in most lung and breast cancer as well as gastrointestinal cells. Its overexpression is linked to liver metastasis, a main reason of death from colorectal cancer [23].

\section{Methods}

\section{Immunization of transgenic rats}

OmniRats ${ }^{\circledR}$ [24-26], transgenic for human antibody variable regions, were immunized by genetic immunization using vaccination vectors encoding for CEACAM5 at Aldevron (Freiburg, Germany), as previously described elsewhere [2]. Experimental procedures and animal care were in accordance with EU animal welfare protection laws and regulations. We confirm that all experimental protocols were approved by a licensing committee from the local government (Landesuntersuchungsamt, Koblenz, Germany).

\section{(See figure on next page.)}

Fig. 1 One step generation of YSD plasmids for the construction of large combinatorial Fab immune libraries using Golden Gate Cloning. Destination plasmids (pDest), entry plasmids ( $\mathrm{pE}$ ) and PCR amplicons contain or are flanked by Bsal recognition sites in different orientations (B: ggtctcn, B: ngagacc). A linear and distinct assembly of those DNA fragments is ensured by the design of complementary signature sequences in defined order within the three modules after Bsal cleavage. a The two-directional (2dir) display system enables the expression of the VH-CH1-Aga2p (Aga2psignal-sequence; SP) gene product under control of the GAL1-promoter whereas the CLC-CLkappa (app8-signal-sequence; App8 SP) gene product is generated under control of the Gal10-promoter. $\mathbf{b}$ The bicistronic display system (bicis) allows for the expression of Fab-fragment heavy and light chains under control of the GAL1-promoter. The generation of distinct VH-CH1-Aga2p (Aga2p-signal-sequence; SP) and CLC-CLkappa (app8-signalsequence; App8 SP) proteins is mediated by ribosomal skipping due to the T2A (2A) peptide. c Schematic illustration of Fab-fragments displayed on the surface of yeast cells. Genes are encoded by a single plasmid and expression is either conducted by two-directional promotors or by ribosomal skipping 


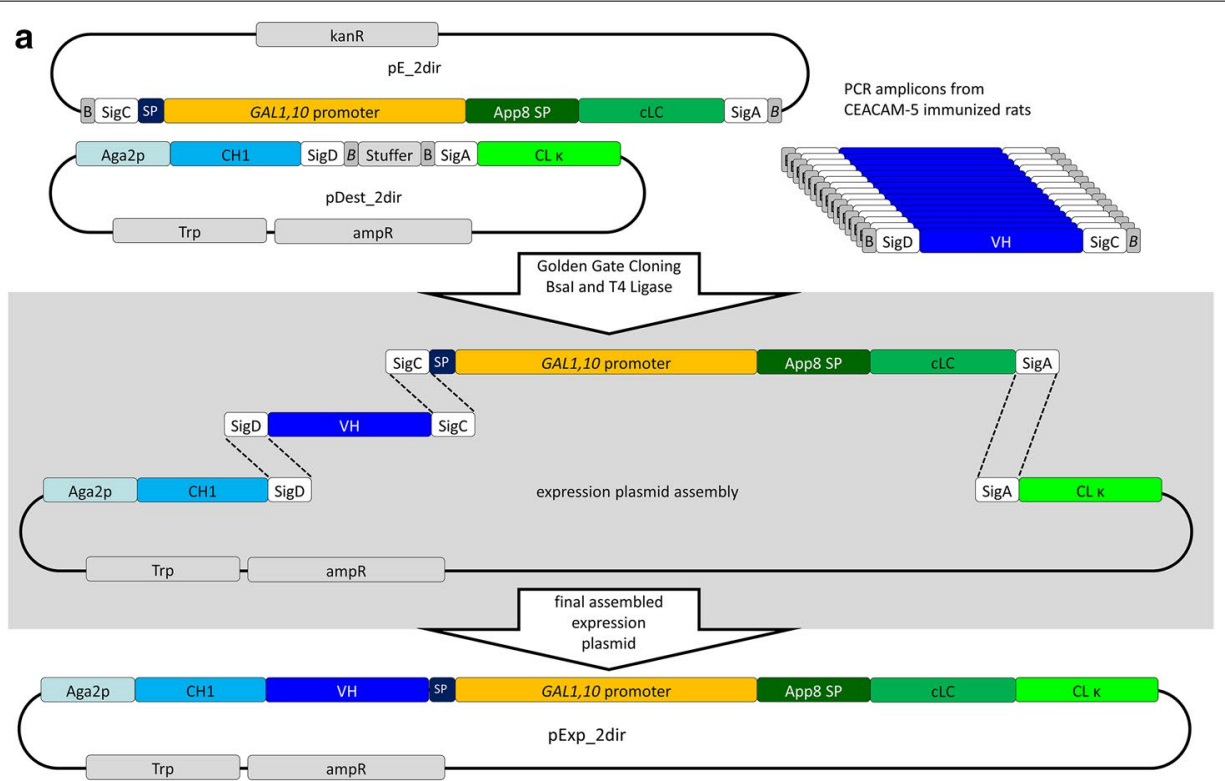

b

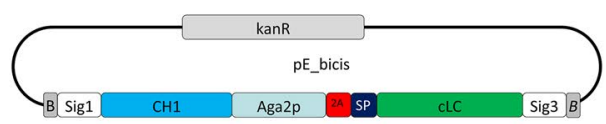
CEACAMp 5 ingom

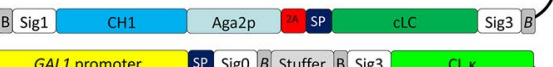

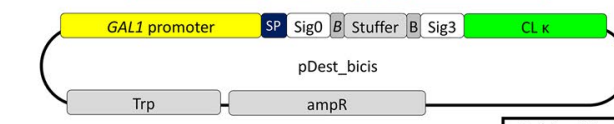
\begin{tabular}{c}
$\begin{array}{c}\text { Golden Gate Cloning } \\
\text { Bsal and T4 Ligase }\end{array}$ \\
\hline
\end{tabular}
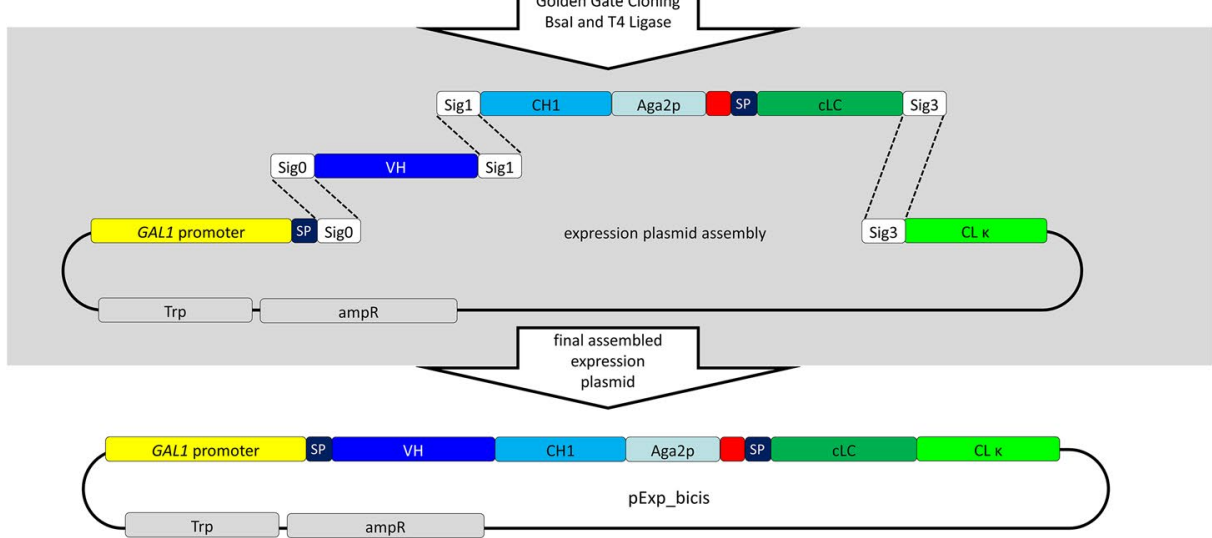

C

Anti Kappa $F\left(a b^{\prime}\right) 2$ PE conjugate Anti His $\mathrm{F}\left(\mathrm{ab} \mathrm{b}^{\prime}\right) 2$ Alexa Flour 647 conjugate Antigen with His-tag

Aga1p

$\mathrm{VH}$

$\mathrm{CH} 1$

G/S-linker + part. Hinge

Aga2p

VL

CL kappa

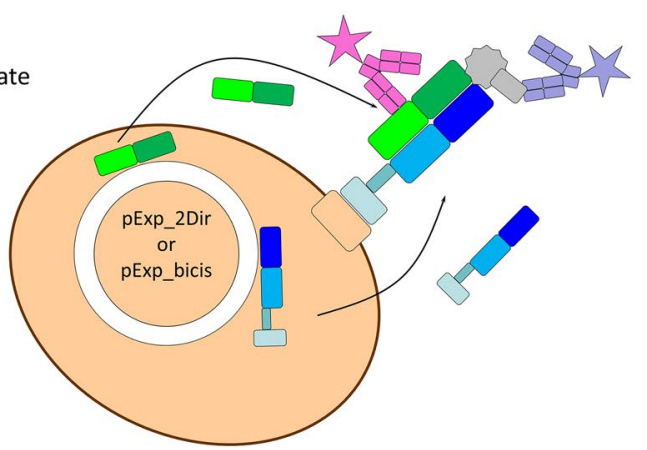




\section{Plasmids}

Plasmids were designed in-house and synthesized at GeneArt (Thermo Fisher Scientific). Genetic elements were derived from pYD1 plasmid backbone (Yeast Display Vector Kit, version D, \#V835-01, Thermo Fisher Scientific) as well as pESC vector series (Agilent). In general, entry plasmids (pEntry) were designed with a kanamycin resistance gene, whereas destination vectors contained an ampicillin resistance cassette as well as a tryptophan selection marker for selection in yeast (Fig. 1).

\section{Yeast strains and media}

For antibody library construction, the Saccharomyces cerevisiae strain EBY100 (MATa URA3-52 trp1 leu $2 \Delta 1$ his3 200 pep4::HIS3 prb1 $1.6 R$ can1 GAL (pIU211:URA3)) was employed (Thermo Fisher Scientific). Initially, EBY100 was cultivated in YPD medium composed of $20 \mathrm{~g} / \mathrm{L}$ peptone, $20 \mathrm{~g} / \mathrm{L}$ dextrose and $10 \mathrm{~g} / \mathrm{L}$ yeast extract supplemented with $10 \mathrm{~mL} / \mathrm{L}$ penicillin/ streptomycin (Gibco). EBY100 cells harboring library plasmids (pDest) after Golden Gate Cloning were cultivated in medium using minimal SD-base (Clontech) with commercially available dropout mix (Clontech) composed of all essential amino acids except for tryptophan, according to the manufacturer's instructions, supplemented with $5.4 \mathrm{~g} / \mathrm{L} \quad \mathrm{Na}_{2} \mathrm{HPO}_{4}$ and $8.6 \mathrm{~g} / \mathrm{L}$ $\mathrm{NaH}_{2} \mathrm{PO}_{4} \times \mathrm{H}_{2} \mathrm{O}$. For induction of antibody gene expression, cells were transferred into respective SG dropout medium wherein glucose was replaced by galactose containing SG-base (Clontech). Additionally, 10\% (w/v) polyethylene glycol 8000 (PEG 8000) was included [27, 28].

\section{Library construction}

Total RNA was extracted from $1 \times 10^{7}$ lymph node cells using RNeasy MiniKit (Qiagen) according to the manufacturer's instructions. For cDNA synthesis $50 \mu \mathrm{L}$ RNA extract, $20 \mu \mathrm{L}$ RT-buffer, $40 \mu \mathrm{L} 25 \mathrm{mM} \mathrm{MgCl} 2,20 \mu \mathrm{L}$ $0.1 \mathrm{M}$ DTT, $10 \mu \mathrm{L}$ RNase Out and $10 \mu \mathrm{L}$ Superscript III reverse transcriptase (SuperScript III First-Strand Kit, Thermo Fisher Scientific) was employed as well as random hexamer primers. Reaction conditions were: $5 \mathrm{~min}$ at $25^{\circ} \mathrm{C}, 60 \mathrm{~min}$ at $50{ }^{\circ} \mathrm{C}$ followed by heat inactivation for $15 \mathrm{~min}$ at $85^{\circ} \mathrm{C}$. Afterwards, $1 \mu \mathrm{L}$ RNase $\mathrm{H}$ was added followed by an incubation step at $37{ }^{\circ} \mathrm{C}$ for $20 \mathrm{~min}$. Human antibody variable regions from OmniRats ${ }^{\circledR}$ were amplified from cDNA in two successive PCR reactions using Q5 High-Fidelity $2 \times$ Master Mix and $50 \mu \mathrm{L}$ reaction volume (NEB). In PCR1, 12 different reactions were prepared with $5 \mu \mathrm{L}$ cDNA using unique forward primers annealing to germline leader sequences and one reverse primer annealing to rat $\mathrm{CH} 1$ domain under the following conditions: $95^{\circ} \mathrm{C}$ for $120 \mathrm{~s}, 30$ cycles of $95^{\circ} \mathrm{C}$ for $15 \mathrm{~s}$, $58{ }^{\circ} \mathrm{C}$ for $30 \mathrm{~s}$ and $72{ }^{\circ} \mathrm{C}$ for $90 \mathrm{~s}$. PCR products were purified via Wizard ${ }^{\circledR}$ SV Gel and PCR Clean-up System (Promega). In the second PCR, human VH domains were amplified with primers incorporating $B s a \mathrm{I}$ recognition sequences for subsequent Golden Gate Cloning. Primers according to Hust et al. [29] were modified and are given in Additional file 1: Table S1. In total for each library design (two-directional display as well as bicistronic display, Fig. 1), nine reactions were performed in parallel using forward primers $1 \mathrm{~S}$ till $9 \mathrm{~S}$ as well as reverse primer 9A. Reaction conditions were as follows: $98^{\circ} \mathrm{C}$ for $30 \mathrm{~s}$, 30 cycles of $98{ }^{\circ} \mathrm{C}$ for $10 \mathrm{~s}, 55^{\circ} \mathrm{C}$ for $20 \mathrm{~s}$ and $72{ }^{\circ} \mathrm{C}$ for $30 \mathrm{~s}$ followed by $72{ }^{\circ} \mathrm{C}$ for $2 \mathrm{~min}$. Afterwards, PCR products were purified via Wizard ${ }^{\circledR}$ SV Gel and PCR Cleanup System (Promega). Finally, PCR products were pooled in an equimolar ratio. For verification of antibody display in both systems, the VH as well as VL of therapeutic antibody trastuzumab [30] were amplified utilizing primers HER2_VH_up and HER2_VH_lo as well as Her2_VL_up and Her2_VL_lo (Additional file 1: Table S1) using the same PCR conditions.

Construction of Fab display libraries was accomplished using Golden Gate Cloning. Reactions were performed in a final volume of $100 \mu \mathrm{L}$ using $1 \mu \mathrm{g}$ of respective destination vector, $2.2 \mu \mathrm{g}$ of the respective entry vector (also encoding for light chain IGKV3-15*01, Fig. 1) and approx. $160 \mathrm{ng}$ of pooled VH PCR product as well as $200 \mathrm{UBsaI}$ (New England Biolabs), 800 U T4 DNA ligase (New England Biolabs) and $10 \mu \mathrm{L} 10 \times \mathrm{T} 4$ Ligase buffer (New England Biolabs). Reaction conditions were 30 cycles of $1 \mathrm{~min}$ at $37^{\circ} \mathrm{C}, 1 \mathrm{~min}$ at $16{ }^{\circ} \mathrm{C}$ followed by $5 \mathrm{~min}$ at $55^{\circ} \mathrm{C}$. After cloning, six reactions were pooled, purified using Wizard ${ }^{\circledR}$ SV Gel and PCR Clean-up System (Promega) and eluted in a final volume of $30 \mu \mathrm{L}$ which were subsequently used for one electroporation reaction into EBY100 as previously described by Benatuil et al. [31]. In general, five transformation reactions were performed for library establishment. Library sizes were calculated by plating out of serial dilutions. For proof of concept display of trastuzumab Fab, Golden Gate settings were slightly modified as follows: $1 \mu \mathrm{g}$ of respective destination vector, $1.4 \mu \mathrm{g}$ of the respective entry vector (Additional file 1: Figure S1), approx. $160 \mathrm{ng}$ of VH PCR product and $160 \mathrm{ng}$ VL PCR product as well as $200 \mathrm{U} \mathrm{BsaI}$ (New England Biolabs), $800 \mathrm{U}$ T4 DNA ligase (New England Biolabs) and $10 \mu \mathrm{L}$ $10 \times$ T4 Ligase buffer (New England Biolabs).

\section{Library screening}

Recombinant human His-tagged CEACAM5 extracellular domain as well as recombinant human His-tagged HER2 extracellular domain were purchased at $R \& D$ systems. For library screening, respective library cells were grown overnight in SD-Trp medium at $30{ }^{\circ} \mathrm{C}$ and $200 \mathrm{rpm}$. Afterwards, cells were transferred to SG-Trp 
medium at approximately $10^{7}$ cells $/ \mathrm{mL}$ followed by incubation at $20{ }^{\circ} \mathrm{C}$ for 2 days. In general, antigen binding was detected by application of Penta-His Alexa Fluor 647 Conjugate antibody (Qiagen, 1:20 diluted in PBS). Fab display on the yeast surface was visualized using light chain specific goat $\mathrm{F}\left(\mathrm{ab}^{\prime}\right)_{2}$ anti-human kappa R-phycoerythrin (SouthernBiotech, 1:20 diluted in PBS). Labeling steps were performed with $10^{7}$ cells $/ 20 \mu \mathrm{L}$ on ice.

Labeling of cells for FACS analysis or sorting was conducted by two consecutive washing steps of library candidates with PBS followed by incubation with the respective antigen at a concentration of $1 \mu \mathrm{M}$. After incubation on ice for approx. $30 \mathrm{~min}$ an additional washing step was performed, followed by staining with Penta-His Alexa Fluor 647 Conjugate antibody as well as light chain specific goat $\mathrm{F}\left(\mathrm{ab}^{\prime}\right)_{2}$ anti-human kappa R-phycoerythrin. Finally, cells were washed with PBS. FACS-sorting rounds were either performed on a Sony SH800 cell sorter (Sony) or on a MoFlo Legacy cell sorter (Beckman Coulter).

\section{Sequencing of enriched yeast cell populations}

Following sorting round three, plasmid DNA of yeast cells was isolated using $1 \mathrm{~mL}$ overnight culture and $\mathrm{RPM}^{\circledR}$ Yeast Plasmid Isolation Kit (MP Biomedicals). Subsequently, obtained plasmids were used for transformation of electrocompetent E. coli Top10 cells (Invitrogen). Afterwards, 96 single clones were randomly chosen and grown overnight in $1 \mathrm{~mL}$ of LB-medium in a 96 deep well plate. The resulting cell suspensions were sent out for sequencing at Microsynth AG (Switzerland) with the following primers 2dir_seq_lo: CAGCAGTACCACCAGATGTAG and bicis_seq_lo: AACTGTTCATCAGATGGTGG.

\section{Expression and purification of isolated library candidates}

VH regions as well as VL IGKV3-15*01 were cloned into pTT5 plasmids, which allow their expression as fulllength IgG molecules in cell culture. Expi293 cells were transiently transfected with expression vectors following the instructions of the manufacturer (Thermo Fisher Scientific). Five days post transfection, antibody containing supernatants were harvested by centrifugation and purified by Antibody Purification Kit and Spin Columns with Prosep-A Media (Merck KGaA). After buffer exchange to PBS using Amicon Ultra-4 Centrifugal Filters (EMD Millipore) full length IgGs were analyzed by SDS-PAGE.

In addition aggregate formation was analyzed by analytical size exclusion chromatography. For this, a TSKgel SuperSW3000 column $(4.6 \times 300 \mathrm{~mm}$, Tosoh Bioscience LLC) and an Agilent HPLC system was used. Differential scanning fluorometry on Prometheus NT.48 (Nanotemper Technologies) was applied to determine thermal stabilities of library candidates.

\section{Biolayer interferometry}

Binding kinetic measurements were performed on the Octet RED96 system (ForteBio, Pall Life Science) at $30{ }^{\circ} \mathrm{C}$ and $1000 \mathrm{rpm}$ agitation (ForteBio, Pall Life Science). Antibodies were loaded on anti-human Fc biosensors (AHC) at $5 \mu \mathrm{g} / \mathrm{mL}$ in PBS for $2 \mathrm{~min}$. Afterwards, tips were transferred to kinetics buffer (KB; PBS, 0.1\% Tween-20 and $1 \%$ bovine serum albumin, BSA) for $60 \mathrm{~s}$ for sensor rinsing. For kinetic analyses, association to CEACAM5 (varying concentrations ranging from 3.125 to $100 \mathrm{nM}$ in KB) was measured for $400 \mathrm{~s}$ followed by dissociation for $900 \mathrm{~s}$ (in KB). In each experiment, one negative control was measured, where the captured antibody was incubated with $K B$ instead of antigen. Data fitting and analysis was performed with ForteBio data analysis software 8.0 using a 1:1 binding model after SavitzkyGolay filtering.

\section{Results}

\section{Design of library components}

In order to investigate whether Golden Gate Cloning allows for library generation in yeast and subsequent isolation of antibodies starting from animal immunization, we decided to adopt a strategy for the isolation of common light chain antibodies which has been previously published by our group [2]. Heavy chain repertoires from immunized transgenic rats were combined with a single light chain. Although the probability of isolating highaffinity antibodies from such libraries is considered to be lower compared to approaches that focus on heavyand light-chain repertoires after animal immunization, it was shown that this is a valid strategy to obtain so-called common light chain antibodies, which might be valuable for the generation of more complex antibody formats such as bispecific antibodies [32].

For library establishment as well as antibody display, two different expression strategies were developed. Both strategies have in common, that for screening of heavy chain diversities with a distinct light chain, three modules are utilized, a destination plasmid, an entry plasmid and the heavy chain variable region module i.e. the PCR amplified $\mathrm{VH}$ repertoire with flanking $B s a \mathrm{I}$ sites and signature sequences allowing for cloning in a defined order (Fig. 1). The two-directional display system (Fig. 1a) is composed of an acceptor plasmid with a tryptophan marker allowing for selection in S. cerevisiae. Major components of this vector for antibody display are the CL-kappa region as well as the $\mathrm{CH} 1$ domain which is fused to Aga2p enabling display on the surface after Golden Gate Cloning (Fig. 1c). Between both components two BsaI sites were introduced and separated by a short stuffer sequence. Signature sequences that allow for one-step cloning were part of CL-kappa (SigA) and CH1 (SigD). Signature sequences 
are given in Additional file 1: Table S2. The entry plasmid contains the GAL1/10 promoters for expression of heavy and light chains, respectively, signal sequences for heavy and light chain secretion as well as a distinct VL (IGKV3$15 * 01)$ [2]. This entry module is flanked by signature sequences SigA as well as SigC of which the latter is incorporated into the signal peptide as well as BsaI sites. PCR amplified $\mathrm{VH}$ repertoires from immunized transgenic rats are flanked with signature sequences (SigC and SigD) as well as $B s a \mathrm{I}$ sites. All restriction sites were designed to be removed during digestion.

The bicistronic display system is composed of a single expression cassette under the control of the GAL1 promoter (Fig. 1b). Simultaneous expression of heavy- and light-chains is enabled via the introduction of the picornaviral $2 \mathrm{~A}$ peptide, mediating ribosomal skipping and therefore the lack of peptide bond formation between Aga2p which is fused to the heavy chain and the light chain. In contrast to internal ribosome entry sites, ribosomal skipping results in the translation of equal amounts of each protein and it has been shown previously that the $2 \mathrm{~A}$ peptide is a versatile tool for yeast surface display [21, 33]. Similarly to the two-directional display system, signature sequences were constituents of sequence-encoding antibody constant regions or signal sequences and BsaI sites were designed to be removed during restriction-ligation reaction, allowing for seamless one-step cloning of the final library display vector (pExp_bicis).

\section{Proof-of-concept: yeast surface display of a HER2-specific Fab}

To validate whether this cloning strategy is valid for antibody YSD in general, we first aimed at displaying the HER2-specific Fab of the therapeutic antibody trastuzumab [30] on the yeast surface using both different vector strategies. A slightly modified cloning scheme was applied, allowing also for the introduction of light chains (Additional file 1: Figure S1). Consequently, entry plasmids were modified in a way that new signature sequences were inserted into the signal peptide mediating light chain expression (SigB for the two-directional system and Sig2 for the bicistronic system). Another entry module, the PCR-amplified VL of trastuzumab was also introduced and flanked with corresponding signature sequences and $B s a \mathrm{I}$ recognition sites. Accordingly, $\mathrm{VH}$ and $\mathrm{VL}$ regions were amplified using primer sets as given in Additional file 1: Table S1. After Golden Gate Cloning, yeast cells were transformed according to Benatuil et al. [31]. Surface presentation of the trastuzumab Fab constructs from single clones of both display systems was characterized by indirect fluorescence labeling of the light chain constant region (CL-kappa) and binding of hexahistidine-tagged HER2 was detected using a fluorescently-labeled Penta-His-specific antibody. As shown in Fig. 2, single clones from both display strategies showed Fab surface expression as well as HER2-binding, clearly demonstrating the functionality of both display systems.

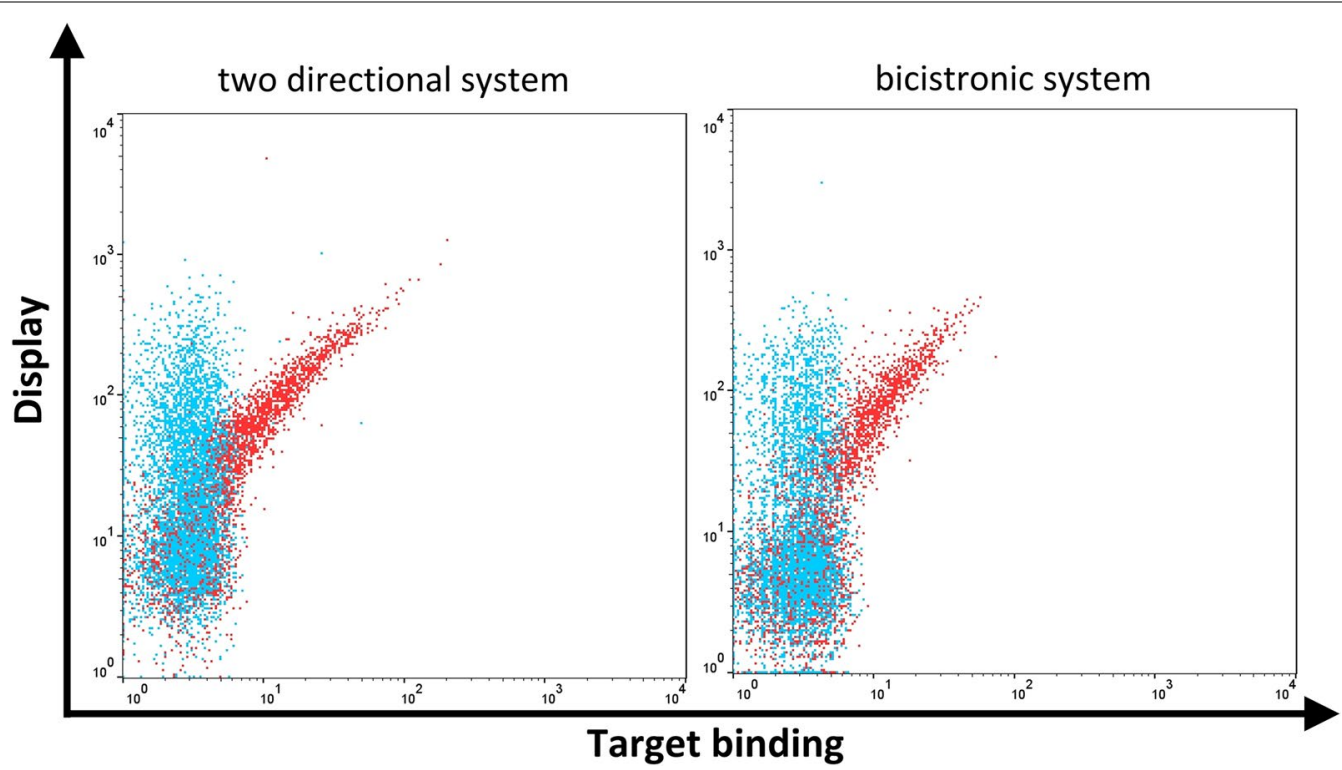

Fig. 2 Overlay of trastuzumab displaying yeast cells either stained with detection antibodies only (blue) or with detection antibodies and HER2 as monitored by flow cytometry. Yeast cells were consecutively incubated with $1 \mu \mathrm{M}$ of His tagged HER2 followed by secondary labeling with Alexa Fluor 647 conjugated anti-Penta-His antibody (target binding) and PE conjugated anti-kappa-antibody (display) 


\section{Library construction and selection of CEACAM5-specific antibodies}

We recently showed that potent CEACAM5-binding Fabs, encompassing a common light chain can be isolated via combination of animal immunization and yeast surface display screening [2]. For library generation via Golden Gate Cloning we applied the same animal-derived lymph nodes for cDNA generation, PCR amplification and eventually for library generation via Golden Gate Cloning. Following cDNA synthesis, heavy chain variable region repertoires were amplified in two consecutive PCR steps. Construction of final display vectors (pExp_2dir, pExp_bicis) was mediated using Golden Gate Cloning. Afterwards, six reactions were pooled and transformed into yeast cells as described by Benatuil et al. [31]. In total, five transformations were performed for each approach resulting in calculated library sizes of $1.3 \times 10^{8}$ (2dir) and $6.9 \times 10^{8}$ (bicis) unique clones, respectively. Sequencing of approximately 100 single clones from each library revealed a high correctness of 95\% (2dir) and 93\% (bicis), i.e. clones that contain functional Fab fragments without frame-shifts or stop-codons. Additionally, no sequence duplets were found in both approaches, indicating high heavy chain diversities of generated libraries.

The two resulting libraries were screened separately by FACS for binders of recombinant human CEACAM5 protein. Target binding was identified by indirect antigen (His-tagged) fluorescence staining with Alexa Fluor 647 conjugated anti-Penta-His antibody. Fab-display levels were analyzed simultaneously by application of kappa light chain specific goat $\mathrm{F}\left(\mathrm{ab}^{\prime}\right)_{2}$ R-PE conjugate. Additionally, controls were introduced, in which respective cells were stained with detection antibodies only (data not shown). These controls were applied to adjust gates in a way that only antigen positive cell populations were considered within the gating strategy. During the first sorting rounds $0.25 \%$ (bicis) and $0.26 \%$ (2dir) double positive events were detected (Fig. 3) and a total of $1.9 \times 10^{8}$ and

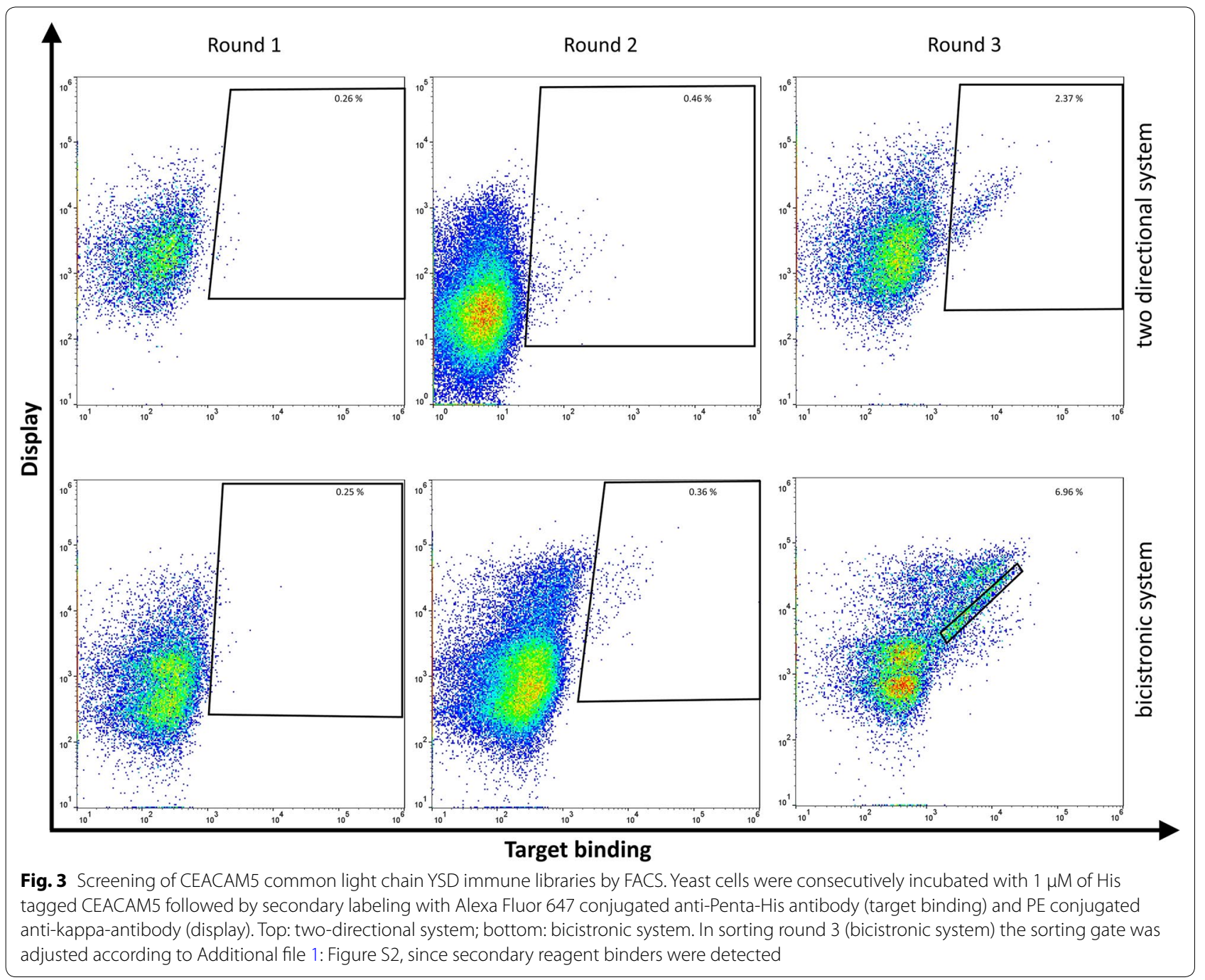


$2 \times 10^{8}$ cells were sorted for each of the different library approaches. Therefore, the theoretical maximum diversity of $1 \times 10^{7}$ variants (lymphocytes used for RNAextraction) was covered approximately 20 -fold. During sorting rounds two and three minimally tenfold output of the previous sorting round was processed (Additional file 1: Table S3). While only a slight enrichment of antigen binding cells was observed in round 2 compared to the first sorting rounds $(0.1-0.2 \%)$, the final FACS-plots (round 3) resulted in 2.4 and $7 \%$ double positives. Interestingly, in the bicistronic approach a significant fraction of cells showed secondary reagent binding against the Alexa Fluor 647 conjugated anti-Penta-His antibody (Fig. 3, Additional file 1: Figure S2). To avoid the isolation of such yeast cells, the sorting gate was adjusted accordingly and only cells were sorted that showed specific binding to CEACAM5.

A final analysis of both approaches after sorting round three demonstrated a distinct population of CEACAM5 binders with a strong correlation between Fab-display and antigen binding (Additional file 1: Figure S3). Finally, 100 single clones from both approaches were sent out for sequencing which revealed that the most frequently emerging $\mathrm{VH}$ sequences were the same in both libraries after sorting (Additional file 1: Figure S4). Interestingly, the two most abundant sequences showing less than 90\% CDR sequence identity (Additional file 1: Figure S4) were also found to be the most prominent clones using the conventional library construction method of separate heavy and light chain vector construction followed by yeast mating [2]. These clones were afterwards reformatted into plasmids which enabled their expression as full length IgG molecules in Expi293 cells. After production and purification via Protein A spin columns, protein purity was analyzed by SEC and thermal stabilities were measured by differential scanning fluorimetry (Additional file 1: Figure S5). Less than 6\% aggregates were determined and thermal stabilities were in the range of about $70{ }^{\circ} \mathrm{C}$ indicating favorable biophysical attributes of isolated library candidates. Moreover, binding kinetics to recombinant CEACAM5 protein were investigated via BLI. Specific high affinity antigen binding in the sub- and single-digit nanomolar range were found for clones from both libraries (Fig. 4), showing clear evidence that both approaches allow for the isolation of antigen-specific antibodies.

\section{Discussion}

Yeast Surface Display has proven to be a versatile technology for antibody engineering and hit discovery [3, 5, $6,11-13,16]$. Antibodies with favorable properties have been isolated from synthetic and naïve repertoires as well as from immunized animals $[2,4,5,11]$. Along with these different sources of diversity, several antibodyformats can be displayed on the surface of yeast cells. While scFv based constructs can be easily expressed from single plasmids [4], a common technique for antibody Fab-display relies on the generation of heavy and light chains plasmids in haploid yeast strains, respectively $[2,5]$. These can be combined by a process referred to as yeast-mating. As a result, the diploid yeast cells display functional Fab fragments on their surface. However, this three-step process of library generation is a sophisticated and laborious procedure.

Taking this into consideration, the present work describes a simplified one-step procedure for the generation of such diversities using Golden Gate Cloning. To demonstrate applicability of this novel system and to ensure comparability, lymph nodes from the same animal were utilized as starting material for library construction as our group already used for the generation of common light chain antibodies [2]. In our previous work, we demonstrated that common light chain antibodies can be isolated from immunized animals using YSD. Libraries were generated using the conventional three-step procedure resulting in a final library size of $2 \times 10^{8}$ individual
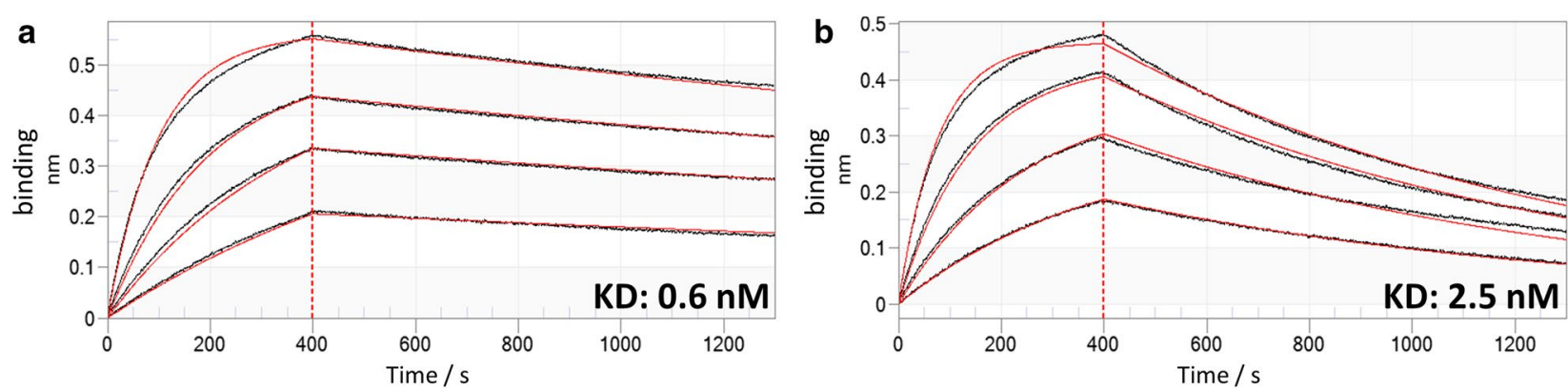

Fig. 4 BLI sensorgrams of kinetic analyses of CEACAM5 binding to immobilized common light chain lgG variants 4G07 (a) and 4F02 (b). Association with recombinant CEACAM5 at concentrations of 25, 12.5, 6.25 and $3.125 \mathrm{nM}$ was measured for $400 \mathrm{~s}$ while dissociation was measured for $900 \mathrm{~s}$. Fitting (red lines) of binding curves (colored lines) was calculated using a 1:1 binding model and Savitzky-Golay filtering 
clones. Interestingly, generation of libraries using Golden Gate Cloning yielded in similar library sizes in a simplified process. Unlike the three-step approach, including construction of antibody chain pools by gap-repair driven homologous recombination and yeast mating that typically takes 2 weeks, the one-step Golden Gate Cloning procedure allows for the introduction of antibody diversities into yeast cells in only 1 week. Furthermore, sequencing of initially established libraries revealed a high diversity of heavy chain variants as well as a high proportion of functional clones, indicating that YSD libraries with suitable qualities can be generated using both Golden Gate Cloning approaches. Three rounds of sorting resulted in a significant enrichment of target binding populations from both libraries (Additional file 1: Figure S3). Interestingly, sequencing of enriched cell pools revealed identical antibody variants as already isolated by Krah et al. (Additional file 1: Figure S4), clearly demonstrating adequate functionality and quality of both display systems in combination with Golden Gate Cloning [2]. However, in the bicistronic display system a significant fraction of cells bound to the AlexaFluor647 conjugated anti-penta-His secondary detection antibody. This might be explained by the fact that antibody diversities derived from Omnirat immunizations were not naturally paired with the utilized common light chain. This may result in novel paratopes showing unspecific binding.

Since the advent of Golden Gate Cloning, as pioneered by Marillonnet et al. in 2008, a plethora of different constructs were genetically engineered [17, 20, 34, 35]. Related to this, this cloning technology was utilized for pathway manipulation in the yeast Yarrowia lipolytica [36]. The Golden Gate Cloning procedure has already been applied for the construction of plasmids used for complex immunological systems, such as the production of $\mathrm{T}$ cell receptor retroviral plasmids for gene transfer into primary $\mathrm{T}$ cells [37]. Additionally, this method has also been used for shuffling libraries for the isolation of protein variants with desired properties [20]. This study demonstrates that Golden Gate Cloning can also be successfully applied to antibody engineering and antibody discovery using YSD.

Within this study, we also aimed at designing and comparing two different display strategies in order to investigate whether one of both systems seems to be better suited for Fab antibody surface display. In the two-directional system, the GAL1/GAL10 promoter is utilized for the expression of the heavy chain and light chain, respectively. Typically, the expression of proteins fused to this promoter system is induced more than 1000-fold by galactose $[38,39]$. Even though it was shown that induction of proteins under the control of the GAL1 promoter result in two- to fourfold higher activity [39], we were able to demonstrate that expression of the heavy chain Fab under the control of the GAL1 promoter and expression of the light chain mediated by the GAL10 promoter result in adequate antibody display levels (Figs. 2, 3).

In the bicistronic display system, a single expression cassette is finally produced under the control of the GAL1 promoter. Equal expression of the heavy chain and the light chain is ensured by the use of the T2A peptide derived from Thosea asigna virus, mediating ribosomal skipping [40]. Since the $2 \mathrm{~A}$ release site is located after the last glycine residue of the peptide sequence, the majority of the peptide is attached to the first protein. We therefore designed the expression cassette in a way that the residual peptide is attached to the C-terminus of Aga2p, since it is known that proteins can be fused at its $\mathrm{C}$ - and $\mathrm{N}$-terminus without perturbing the functional integrity of Aga2p $[2,6]$. Similarly, the last proline residue of the 2A peptide is the first residue of the second protein that is being translated, which was either the Aga2p signal peptide in the bicistronic library approach or the App8 signal peptide for the bicistronic display of HER2-targeting trastuzumab [41]. Consequently, this proline residue is not part of the finally processed and secreted light chain. Additionally, we were able to show that display levels were quite similar between both display systems, indicating that the proline residue does not negatively impact processing of both signal peptides. Ultimately, both display systems allowed for the generation of large antibody Fab libraries with similar unique clone numbers. Likewise also the isolation of identical target-specific antibodies proves that both approaches seem to be applicable YSD systems for antibody discovery and engineering. As both technologies allow the incorporation of antibody diversities into destination plasmids in a single step and identical binders were derived from both selection campaigns, a recommendation for either one of the display technologies based on the presented data cannot be given.

In this work, the light chain sequence was kept constant throughout the cloning and screening procedure since we aimed at isolating common light chain antibodies, where in theory antigen binding is mainly or exclusively mediated by the heavy chain [2]. Since large library sizes exceeding $10^{8}$ variants can easily be obtained, the Golden Gate Cloning strategies presented here may also be amenable to the simultaneous generation and combination of VH and VL repertoires. Notably, this could significantly simplify the isolation of conventional antibodies from immunized animals. 


\section{Conclusion}

The generation of antibody Fab immune libraries for YSD is a tedious and time consuming multistep process which includes the generation of heavy and light chain diversities in haploid yeast cells that need to be combined via yeast mating. In this work we describe a simplified procedure for the generation of such libraries based on Golden Gate Cloning. By application of this technology, combinatorial libraries can be easily constructed in just one single step.

The feasibility of the presented approach was demonstrated in a proof of concept study, in which large Fab repertoires were generated and high affinity common light chain antibodies were identified after three rounds of FACS utilizing two different antibody display approaches. According to our findings, this process might also be applicable for the random generation and combination of $\mathrm{VH}$ and VL diversities, which would significantly improve the antibody hit discovery process from immunized rodents.

\section{Additional file}

Additional file 1. Additional tables and figures.

\begin{abstract}
Abbreviations
Bicis: bicistronic display system; BLI: biolayer interferometry; CDR: complementarity determining region; pDest: destination plasmid; DSF: differential scanning fluorimetry; pE: entry plasmid; pExp: expression plasmid; FACS: fluorescence activated cell sorting; 2 dir: two-directional display system; SEC: size exclusion chromatography; $\mathrm{VH}$ : variable domain of the heavy chain; $\mathrm{VL}$ : variable domain of the light chain; YSD: yeast surface display.
\end{abstract}

\section{Authors' contributions}

SR, HK, SZ, SK designed the experiments. SR, JG, BV, DD, IW, RG, SZ, SK performed the experiments. SB, LT, JG, BV, HK, SZ, SK analyzed the experiments. $H K, S Z, S K$ wrote the manuscript. All authors read and approved the final manuscript.

\section{Author details}

${ }^{1}$ Institute for Organic Chemistry and Biochemistry, Technische Universität Darmstadt, Alarich-Weiss-Strasse 4, 64287 Darmstadt, Germany. ${ }^{2}$ Protein Engineering and Antibody Technologies, Merck KGaA, Frankfurter Strasse 250, 64293 Darmstadt, Germany. ${ }^{3}$ Chemical and Pharmaceutical Development, Merck KGaA, Frankfurter Straße 250, 64293 Darmstadt, Germany.

\section{Acknowledgements}

We want to thank Tanja Lehmann and Kerstin Hallstein for experimental support.

\section{Competing interests}

The authors declare that they have no competing interests.

\section{Availability of data and materials}

All data generated or analyzed during this study are included in this published article (and its additional file).

\section{Ethics approval and consent to participate}

We confirm that all experimental protocols were approved by a licensing committee from the local government (Landesuntersuchungsamt, Koblenz, Germany).
Funding

Not applicable.

\section{Publisher's Note}

Springer Nature remains neutral with regard to jurisdictional claims in published maps and institutional affiliations.

Received: 9 August 2017 Accepted: 20 December 2017

Published online: 09 January 2018

\section{References}

1. Boder ET, Wittrup KD. Yeast surface display for screening combinatorial polypeptide libraries. Nat Biotechnol. 1997;15:553-7.

2. Krah S, Schröter C, Eller C, Rhiel L, Rasche N, et al. Generation of human bispecific common light chain antibodies by combining animal immunization and yeast display. Protein Eng Des Sel. 2017;30:291-301.

3. Schröter C, Günther R, Rhiel L, Becker S, Toleikis L, et al. A generic approach to engineer antibody $\mathrm{pH}$-switches using combinatorial histidine scanning libraries and yeast display. MAbs. 2015;7:138-51.

4. Wang B, Lee $\mathrm{CH}$, Johnson EL, Kluwe CA, Cunningham JC, Tanno H, et al. Discovery of high affinity anti-ricin antibodies by $B$ cell receptor sequencing and by yeast display of combinatorial $\mathrm{VH}: \mathrm{VL}$ libraries from immunized animals. MAbs. 2016;25:1-10.

5. Weaver-Feldhaus JM, Lou J, Coleman JR, Siegel RW, Marks JD, Feldhaus MJ. Yeast mating for combinatorial Fab library generation and surface display. FEBS Lett. 2004;564:24-34.

6. Zielonka S, Weber N, Becker S, Doerner A, Christmann A, et al. Shark attack: high affinity binding proteins derived from shark vNAR domains by stepwise in vitro affinity maturation. J Biotechnol. 2014;191:236-45.

7. Doerner A, Rhiel L, Zielonka S, Kolmar H. Therapeutic antibody engineering by high efficiency cell screening. FEBS Lett. 2014;588:278-87.

8. Könning D, Zielonka S, Sellmann C, Schröter C, Grzeschik J, et al. Isolation of a $\mathrm{pH}$-sensitive IgNAR variable domain from a yeast-displayed, histidine-doped master library. Mar Biotechnol (NY). 2016;18:161-7.

9. Traxlmayr MW, Faissner M, Stadlmayr G, Hasenhindl C, Antes B, et al. Directed evolution of stabilized lgG1-Fc scaffolds by application of strong heat shock to libraries displayed on yeast. Biochim Biophys Acta. 2012;1824:542-9.

10. TraxImayr MW, Lobner E, Hasenhindl C, Stadlmayr G, Oostenbrink C, et al. Construction of $\mathrm{pH}$-sensitive Her2-binding lgG1-Fc by directed evolution. Biotechnol J. 2014;9:1013-22.

11. Feldhaus MJ, Siegel RW, Opresko LK, Coleman JR, Feldhaus JM, et al. Flowcytometric isolation of human antibodies from a nonimmune Saccharomyces cerevisiae surface display library. Nat Biotechnol. 2003;21:163-70.

12. Wozniak-Knopp G, Bartl S, Bauer A, Mostageer M, Woisetschlager M, et al. Introducing antigen-binding sites in structural loops of immunoglobulin constant domains: FC fragments with engineered HER2/neu-binding sites and antibody properties. Protein Eng Des Sel. 2010;23:289-97.

13. Zielonka S, Empting M, Könning D, Grzeschik J, Krah S, et al. The shark strikes twice: hypervariable loop 2 of shark IgNAR antibody variable domains and its potential to function as an autonomous paratope. Mar Biotechnol (NY). 2015;17:386-92.

14. Glotzbach B, Reinwarth M, Weber N, Fabritz S, Tomaszowski M, et al. Combinatorial optimization of cystine-knot peptides towards high-affinity inhibitors of human matriptase-1. PLOS ONE. 2013;8:e76956.

15. Maass F, Wüstehube-Lausch J, Dickgiesser S, Valldorf B, Reinwarth M, et al. Cystine-knot peptides targeting cancer-relevant human cytotoxic $T$ lymphocyte-associated antigen 4 (CTLA-4). J Pept Sci. 2015;21:651-60.

16. Rhiel L, Krah S, Günther R, Becker S, Kolmar H, Hock B. REAL-Select: fulllength antibody display and library screening by surface capture on yeast cells. PLoS ONE. 2014;9:e114887.

17. Engler C, Kandzia R, Marillonnet S. A one pot, one step, precision cloning method with high throughput capability. PLOS ONE. 2008;3:e3647.

18. Lee JH, Skowron PM, Rutkowska SM, Hong SS, Kim SC. Sequential amplification of cloned DNA as tandem multimers using class-IIS restriction enzymes. Genet Anal. 1996;13:139-45. 
19. Padgett KA, Sorge JA. Creating seamless junctions independent of restriction sites in PCR cloning. Gene. 1996;168:31-5.

20. Engler C, Gruetzner R, Kandzia R, Marillonnet S. Golden gate shuffling: a one-pot DNA shuffling method based on type lls restriction enzymes. PLOS ONE. 2009:4:e5553.

21. Grzeschik J, Hinz SC, Könning D, Pirzer T, Becker S, et al. A simplified procedure for antibody engineering by yeast surface display: coupling display levels and target binding by ribosomal skipping. Biotechnol J. 2017. https://doi.org/10.1002/biot.201600454.

22. van den Beucken T, Pieters H, Steukers M, van der Vaart M, Ladner RC, et al. Affinity maturation of Fab antibody fragments by fluorescent-activated cell sorting of yeast-displayed libraries. FEBS Lett. 2003;546:288-94.

23. Lee JH, Lee SW. The roles of carcinoembryonic antigen in liver metastasis and therapeutic approaches. Gastroenterol. Res. Pract. 2017;2017:7521987.

24. Bruggemann M, Osborn MJ, Ma B, Hayre J, Avis S, et al. Human antibody production in transgenic animals. Arch Immunol Ther Exp (Warsz). 2015:63:101-8.

25. Osborn MJ, Ma B, Avis S, Binnie A, Dilley J, et al. High-affinity lgG antibodies develop naturally in Ig-knockout rats carrying germline human IgH/lgkappa/Iglambda loci bearing the rat $\mathrm{CH}$ region. J Immunol. 2013:190:1481-90.

26. Osbourn J, Groves M, Vaughan T. From rodent reagents to human therapeutics using antibody guided selection. Methods. 2005;36:61-8.

27. Arnold K, Herrmann A, Pratsch L, Gawrisch K. The dielectric properties of aqueous solutions of poly(ethylene glycol) and their influence on membrane structure. Biochim Biophys Acta. 1985;815:515-8.

28. Uma Maheswar Rao JL, Satyanarayana T. Enhanced secretion and low temperature stabilization of a hyperthermostable and $\mathrm{Ca} 2+-$ independent alpha-amylase of Geobacillus thermoleovorans by surfactants. Lett Appl Microbiol. 2003;36:191-6.

29. Kügler J, Wilke S, Meier D, Tomszak F, Frenzel A, et al. Generation and analysis of the improved human HAL9/10 antibody phage display libraries. BMC Biotechnol. 2015;15:1.

30. Reichert JM. Taylor \& Francis, 2012. p. 413-415.
31. Benatuil L, Perez JM, Belk J, Hsieh CM. An improved yeast transformation method for the generation of very large human antibody libraries. Protein Eng Des Sel. 2010;23:155-9.

32. Krah S, Sellmann C, Rhiel L, Schröter C, Dickgiesser S, et al. Engineering bispecific antibodies with defined chain pairing. N Biotechnol. 2017;39:167-73.

33. Cruz-Teran CA, Tiruthani K, Mischler A, Rao BM. Inefficient ribosomal skipping enables simultaneous secretion and display of proteins in Saccharomyces cerevisiae. ACS Synth Biol. 2017;6:2096-107.

34. Engler C, Youles M, Gruetzner R, Ehnert TM, Werner S, et al. A golden gate modular cloning toolbox for plants. ACS Synth Biol. 2014;3:839-43.

35. Luo Y, Lin L, Bolund L, Sorensen CB. Efficient construction of rAAVbased gene targeting vectors by Golden Gate Cloning. Biotechniques. 2014;56:263-8

36. Celinska E, Ledesma-Amaro R, Larroude M, Rossignol T, Pauthenier C, Nicaud JM. Golden gate assembly system dedicated to complex pathway manipulation in Yarrowia lipolytica. Microb Biotechnol. 2017;10:450-5.

37. Coren LV, Jain S, Trivett MT, Ohlen C, Ott DE. Production of retroviral constructs for effective transfer and expression of T-cell receptor genes using Golden Gate Cloning. Biotechniques. 2015;58:135-9.

38. Johnston M, Davis RW. Sequences that regulate the divergent GAL1GAL10 promoter in Saccharomyces cerevisiae. Mol Cell Biol. 1984:4:1440-8.

39. Yocum RR, Hanley S, West R Jr, Ptashne M. Use of lacZ fusions to delimit regulatory elements of the inducible divergent GAL1-GAL10 promoter in Saccharomyces cerevisiae. Mol Cell Biol. 1984;4:1985-98.

40. Ryan MD, King AM, Thomas GP. Cleavage of foot-and-mouth disease virus polyprotein is mediated by residues located within a 19 amino acid sequence. J Gen Virol. 1991;72(Pt 11):2727-32.

41. Rakestraw JA, Sazinsky SL, Piatesi A, Antipov E, Wittrup KD. Directed evolution of a secretory leader for the improved expression of heterologous proteins and full-length antibodies in Saccharomyces cerevisiae. Biotechnol Bioeng. 2009;103:1192-201.

\section{Submit your next manuscript to BioMed Central and we will help you at every step:}

- We accept pre-submission inquiries

- Our selector tool helps you to find the most relevant journal

- We provide round the clock customer support

- Convenient online submission

- Thorough peer review

- Inclusion in PubMed and all major indexing services

- Maximum visibility for your research

Submit your manuscript at www.biomedcentral.com/submit
() Biomed Central 